\title{
Optical Trapping of Nanoparticles with Plasmonic Apertures Generated by Algorithm
}

\author{
Neuton Li ${ }^{1,2}$, Jasper Cadusch ${ }^{3}$, Kenneth B. Crozier ${ }^{2,3,4}$ \\ 1 - Research School of Physics, Australian National University, Australian Capital Territory 2600 Australia \\ 2 - School of Physics, University of Melbourne, Victoria 3010 Australia \\ 3 - Department of Electrical and Electronic Engineering, University of Melbourne, Victoria 3010 Australia \\ 4 - ARC Centre of Excellence for Transformative Meta-Optical Systems (TMOS), University of Melbourne, Victoria 3010 Australia \\ neuton.li@anu.edu.au kcrozier@unimelb.edu.au
}

\begin{abstract}
Plasmonic apertures for optical nanotweezers are designed by an algorithm and fabricated with a helium ion microscope. Optical trapping experiments are performed. At every laser intensity, an algorithm-designed structure can outperform a conventional plasmonic aperture. (C) 2020 The Author(s)
\end{abstract}

\section{Introduction}

The ability to optically trap nanoparticles at ever lower laser powers would be advantageous for the field of nanoscience. To produce strong trapping potentials, the gradient force can be boosted by concentrating light as tightly as possible at the trapping site [1], e.g. with optical nanostructures. Plasmonic apertures have proven effective, with their enhanced fields producing strong gradient forces. Various aperture shapes have been investigated, including circular and rectangular nanoholes [2], coaxial apertures [3], bowtie apertures [4], C-shaped apertures [5], and double nanoholes ( $\mathrm{DNH},[6])$. We have previously shown by simulation the opportunities presented by algorithm-designed apertures [7]. Here, we report experimental results. At every laser intensity, the algorithm-designed structures trap particles more tightly than a nanoaperture that has been widely employed for optical nanotweezers, the double nanohole.

\section{Design and Fabrication}

The designs we investigate are the simulated annealing aperture (SA) from Ref. [7], another aperture (SA2) also optimized by simulated annealing, and a double nanohole (DNH). The aperture SA2 is designed by optimizing for the shape that increases the force $\mathbf{F}_{\mathbf{z}}$ on a particle $(10 \mathrm{~nm}$ diameter, $\mathrm{n}=1.6)$ at the aperture entrance. As the simulated annealing algorithm progresses (Fig 1a), $\mathbf{F}_{\mathbf{z}}$ increases from its starting value of $0.5 \mathrm{fN}$ for the starting design $(\mathrm{DNH})$ to be $>3 \mathrm{fN}$ for the final design ("SA2"). The simulations are performed in water with a focused beam $(\lambda=1064 \mathrm{~nm}, \mathrm{NA}=1.3)$ whose peak intensity is $6.67 \mathrm{~mW} / \mu \mathrm{m}^{2}$. Simulations of the SA and SA2 structures show that they offer similar trapping performance. We also use the algorithm to design a structure we term a ring coupler. This consists of a pair of concentric rings and can further increase the electric field enhancement (and the force). We use a helium ion microscope (HIM) to etch the apertures into a gold film on a glass substrate. We produce the different aperture designs (DNH, SA, SA2) with and without ring couplers (Fig 1b-e). The ring couplers are milled with a neon ion beam.

\section{Measurement Results, Discussion and Conclusion}

We use an inverted microscope equipped with an infrared laser $(\lambda=1064 \mathrm{~nm})$ for trapping and a green laser for fluorescence excitation. This allows direct observation of individual trapped nanoparticles via fluorescence microscopy with an electron-multiplying charge coupled device (EMCCD). We extract the particle position in the $x-y$ plane from each movie frame for trapping with the DNH aperture, the SA aperture, and the SA2 aperture with ring coupler. We separate these distributions into orthogonal components (i.e. along $\mathrm{x}$ - and $\mathrm{y}$-axes), allowing us to find the effective trapping stiffness along these axes. The trapping stiffnesses of the SA + ring and SA2 + ring apertures are much greater than that of the DNH for particles with diameters of $100 \mathrm{~nm}$ (Fig 2a, b) and $27 \mathrm{~nm}$ (Fig 2c, d), at every laser intensity. Laser intensity thresholds exist for trapping with the $\mathrm{DNH}$, below which trapping is not observed. These are $\sim 2.5$ $\mathrm{mW} / \mu \mathrm{m}^{2}$ for $100 \mathrm{~nm}$ diameter particles and $\sim 6 \mathrm{~mW} / \mu \mathrm{m}^{2}$ for $27 \mathrm{~nm}$ diameter particles. Our results demonstrate that the trapping performance of the algorithm-optimized structures outdo that of the DNH. 
In summary, we demonstrated by simulation and experiment that algorithm-designed plasmonic apertures surpass the performance of a conventional aperture, the DNH. To the best of our knowledge, this work renresents the firgt exnerimental demonstration of optical nanotweezers designed by algorithm.

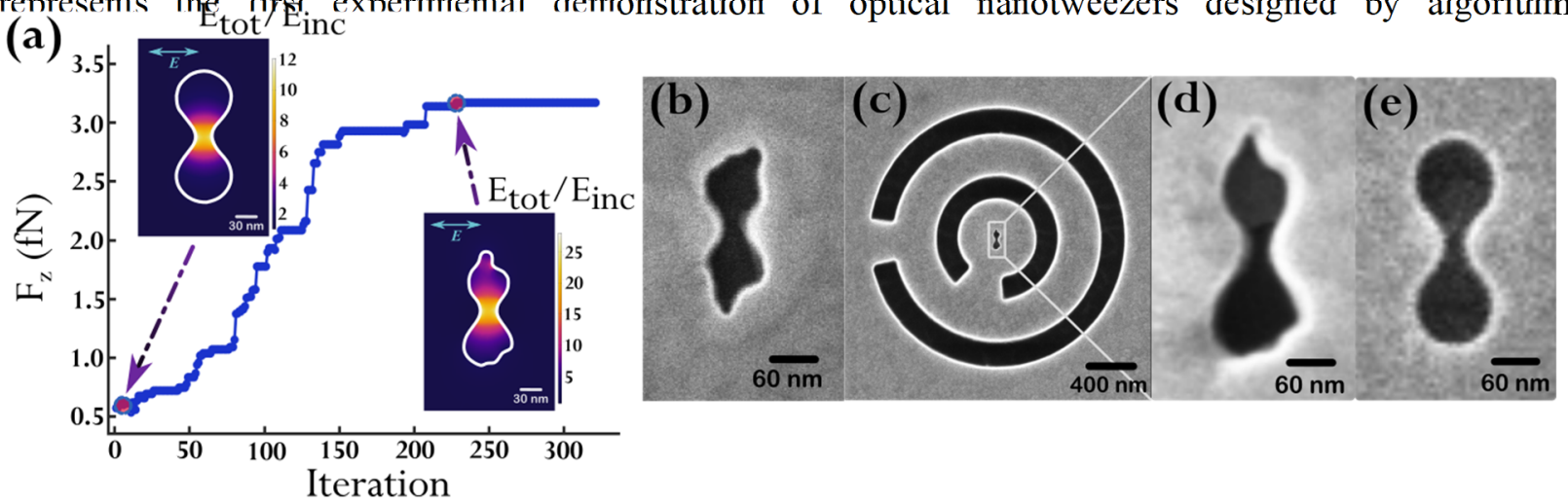

Fig 1: (a) Force on nanoparticle near aperture entrance $\left(\mathbf{F}_{\mathbf{z}}\right)$ vs iteration step of simulated annealing algorithm. SEM images of etched devices in gold showing (b) SA, (c) SA2 + ring, (d) zoom in of SA2, and (e) DNH apertures.
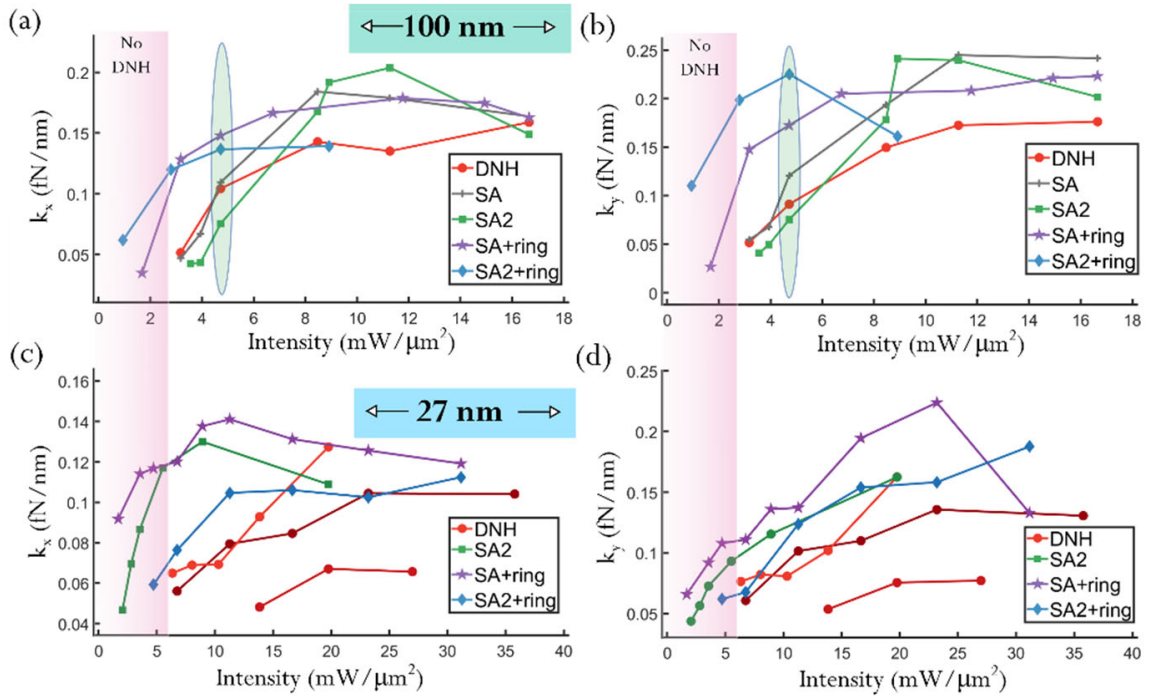

Fig 2: Experimentally estimated equivalent trap stiffnesses for (a-b) $100 \mathrm{~nm}$ diameter particle in $\mathrm{x}$ and y directions, respectively, and for (c-d) $27 \mathrm{~nm}$ diameter particle in $\mathrm{x}$ and y directions, respectively.

\section{References}

[1] D. Gao et al., "Optical manipulation from the microscale to the nanoscale: fundamentals, advances and prospects," Light Sci. \&Amp; Appl. 6, 17039-(2017)

[2] E.-S. Kwak et al., "Optical Trapping with Integrated Near-Field Apertures," J. Phys. Chem. B 108, 13607 (2004)

[3] A. A. E. Saleh and J. A. Dionne, "Toward Efficient Optical Trapping of Sub-10-nm Particles with Coaxial Plasmonic Apertures," Nano Lett. 12, 5581 (2012)

[4] R. A. Jensen et al., "Optical Trapping and Two-Photon Excitation of Colloidal Quantum Dots Using Bowtie Apertures," ACS Photonics 3, 423 (2016)

[5] M. A. Zaman, P. Padhy, and L. Hesselink, "Near-field optical trapping in a non-conservative force field," Sci. Rep. 9, 649 (2019)

[6] Y. Pang and R. Gordon, "Optical Trapping of $12 \mathrm{~nm}$ Dielectric Spheres Using Double-Nanoholes in a Gold Film," Nano Lett. 11, 3767 (2011)

[7] N. Li, J. Cadusch, and K. Crozier, "Algorithmic approach for designing plasmonic nanotweezers," Opt. Lett. 44, $5250(2019)$ 


\section{University Library}

\section{- M M I N E R VA A gateway to Melbourne's research publications}

Minerva Access is the Institutional Repository of The University of Melbourne

Author/s:

Li, N;Cadusch, J;Crozier, KB

Title:

Optical trapping of nanoparticles with plasmonic apertures generated by algorithm

Date:

2021-01-01

Citation:

Li, N., Cadusch, J. \& Crozier, K. B. (2021). Optical trapping of nanoparticles with plasmonic apertures generated by algorithm. Optics InfoBase Conference Papers, pp.fw3m.3-. Optica Publishing Group. https://doi.org/10.1364/cleo_qels.2021.fw3m.3.

Persistent Link:

http://hdl.handle.net/11343/294862 